\title{
1 Prevalence of feline lungworm Aelurostrongylus abstrusus in England
}

2

3 Hany M. Elsheikha ${ }^{1}$, Ian Wright ${ }^{2}$, Bo Wang ${ }^{3}$, Roland Schaper ${ }^{4}$

4

5

$6 \quad{ }^{1}$ School of Veterinary Medicine and Science, University of Nottingham, LE12 5RD, UK

$7 \quad{ }^{2}$ The Mount Veterinary Practice, 1 Harris Str, Fleetwood Lancs, FY7 6QX, UK

$8{ }^{3}$ Department of Mathematics, University of Leicester, Leicester LE1 7RH, UK

$9 \quad{ }^{4}$ Bayer Animal Health GmbH, 51368 Leverkusen, Germany

10

11 *Corresponding author. Tel: +44 1159516445; fax: +44 1159516440;

12 E-mail address: hany.elsheikha@nottingham.ac.uk (H.M. Elsheikha)

13

14

15

16

17

18

19

20

21

22

23

24

25 


\section{Abstract}

Infection of cats with lungworm Aelurostrongylus abstrusus has recently been documented in the UK. Here, we aimed to study the prevalence of $A$. abstrusus in fecal samples from cats across England. A total of 950 fecal samples were collected from cats together with information on their age, breed, gender, geographic region, lifestyle, and treatment history. A total of 17 (1.7\%) cats were positive for A. abstrusus based on species-specific morphological features of the larvae isolated by Baermann's technique. There was no statistically significant difference in the proportion of positive samples between females $(506 ; 53.2 \%)$ and males $(444 ; 46.7 \%)$. Multiple regression analysis showed that prevalence of feline lungworm was significantly different across geographic regions: in comparison with East Midlands, some regions had shown significantly increased odds of $A$. abstrusus-positive samples (South East [odds ratio $[\mathrm{OR}]=7.68 ; 95 \%$ confidence interval $[\mathrm{CI}]=1.70$ to $32.76 ; p=0.01]$; West Midlands $[\mathrm{OR}=$ $6.20 ; 95 \% \mathrm{CI}=1.21$ to $26.84 ; p=0.02]$ ), while other regions had also increased odds although not statistically significant (Greater London $[\mathrm{OR}=9.63 ; 95 \% \mathrm{CI}=0.43$ to $84.05 ; p=0.07]$; North West $[\mathrm{OR}=4.25 ; 95 \% \mathrm{CI}=0.59$ to $20.89 ; p=0.09]$; South West $[\mathrm{OR}=2.48 ; 95 \% \mathrm{CI}$ $=0.12$ to $17.64 ; p=0.43]$; and North East $[\mathrm{OR}=1.88 ; 95 \% \mathrm{CI}=0.10$ to $12.24 ; p=0.57])$. Keeping cats inside was protective against the risk of infection compared with those having outdoor access $(\mathrm{OR}=0.09 ; 95 \% \mathrm{CI}=0.01$ to $0.48 ; p=0.02)$. On the other hand, age, breed, gender and deworming history did not have any significant effect on the likelihood of infection.

Our data indicate that $A$. abstrusus is a parasite of potential significance in cats, in particular those from certain geographic regions in England. To reduce the spread of this parasite, an integrated feline lungworm control program needs to be implemented.

Keywords: Cats; lungworm; Aelurostrongylus abstrusus; prevalence; survey; risk factors 


\section{Introduction}

The gastropod-borne nematode Aelurostrongylus abstrusus (Railliet, 1898) is the most common lungworm of domestic and wild felids, and is found in many parts of the world, including Europe, USA, South America and Australia (Scott, 1973; Elsheikha et al., 2016; Giannelli et al., 2017; Penagos-Tabares et al., 2018). This parasite has a considerable impact on the health and welfare of cats. Also, it has shown both regional endemicity and geographic expansion across Europe. Infected cats exhibit chronic wasting, cough, dyspnea, pulmonary wheezes and other signs of lower airway disease, although asymptomatic cases, shedding high number of larvae in feces, may also occur (Genchi et al., 2014; Elsheikha et al., 2016; Hansen et al., 2017). In addition to $A$. abstrusus, recent studies have detected other metastrongyloids, such as Troglostrongylus brevior (Crenosomatidae) and Oslerus rostratus (Filaroididae) and the trichurid Eucoleus aerophilus (syn. Capillaria aerophila) in the lungs of cats (Pennisi et al., 2015; Giannelli et al., 2017).

Biological and epidemiological drivers (Traversa et al., 2009; Beugnet et al., 2014; Hansen et al., 2017), some of them yet unconfirmed, appear to be increasing the risk of infection in cats in certain parts of the world. However, important gaps remain in the available literature surrounding the prevalence of feline lungworm infection and its epidemiological patterns as well as determinants. Lack of understanding of these changing patterns may have serious implications from a clinical standpoint, given that a delay in diagnosis and treatment can lead to severe lesions and even death of the infected cat. Recently, more cases have begun to be observed by clinicians (Gunn-Moore and Elsheikha, 2018). Despite this increasing frequency of A. abstrusus in cats, there is lack of epidemiological studies that assess the prevalence and distribution of this parasite in cats in The UK. A pan-European study involving 12 countries, reported $0 \%$ A. abstrusus infection rate in fecal samples collected from Cambridge, UK (Giannelli et al., 2017). However, this study is not representative to the status of A. abstrusus 
infection in The UK due to its very small sample size. Given the paucity of data on A. abstrusus in The UK, a larger survey involving more samples collected from diverse geographic areas is needed in order to provide important insight into the transmission potential of A. abstrusus in cats.

We previously conducted a cross-sectional survey in England and in the initial phase we detected A. abstrusus larvae in the feces of 2.2\% (14 out of 629) of cats (Elsheikha et al., 2017). Herein, we report a more up-to-date $A$. abstrusus prevalence rate, after the completion of the survey, based on the analysis of 950 fecal samples from cats across seven main geographic regions of England. Our study established a new background prevalence of $A$. abstrusus in cats in England and identified outdoor access as a potential risk factor for A. abstrusus infection. This new knowledge may lead to more insight into the real burden and risk of feline lungworm infection in the UK, which will ultimately lead to improved sustainable management strategies for feline aelurostrongylosis.

\section{Materials and methods}

\subsection{Fecal samples and data collection}

From January 2016 to January 2018, fecal samples $(n=950)$ were collected from cats, 506 females and 444 males, across seven administrative regions of England. The study was designed to include feral and street cats in addition to domestic cats. Fecal samples were collected from cats from shelters, catteries and privately owned cats, and were examined using Baermann's technique in order to isolate the first stage larvae (L1s). Morphological identification of the isolated A. abstrusus L1s and its differentiation from L1s of other metastrongyloids was achieved via microscopic examination using previously described 
101 morphometric features of A. abstrusus larvae (Gerichter, 1949; Brianti et al., 2014; Giannelli et al., 2014; Giannelli et al., 20117).

Data on age (kitten [0 - 6 months]; junior [7 months -2 years]; prime [3 years -6 years]; mature [ 7 years - 10 years]; and senior [11 years - 14 years]), breed, gender (male vs female), main geographic regions in England (North East, North west, South East, South West, East Midlands, West Midlands, and Greater London), lifestyle (indoor, outdoor access, feral, and stray) and deworming history (recently treated using anthelmintics, such as emodepside $\left[\right.$ Profender ${ }^{\circledR}$ ] or macrocyclic lactones, which have reported efficacy against A. abstrusus vs left un-treated) were collected. Ethical approval was granted by the Research Ethics Committee of

110 School of Veterinary Medicine and Science, University of Nottingham.

\subsection{Prevalence and risk factor analyses}

113 Statistical relationships were assessed between fecal shedding of $A$. abstrusus larvae and

114 defined risk factors, such as age, breed, gender, geographic location, cat lifestyle, and animal

115 treatment status at the time of fecal sampling. The overall parasite prevalence was determined

116 by dividing the number of parasite-positive fecal samples by the total number of samples

117 collected within each risk factor category. Test of independence for contingency tables was

118 used to evaluate associations between each risk factor (e.g., animal age, breed, gender etc.) and

119 presence of $A$. abstrusus larvae. Multiple logistic regression was used to investigate the 120 associations between host-specific, demographic, and environmental risk factors with respect

121 to test outcome (e.g., parasite present or absent). Risk factors that were significant at a $p$ level 122 of $<0.1$ were then incorporated in a forward-stepping manner into multiple logistic regression

123 models. These multivariable models yielded adjusted odds ratios (OR) that simultaneously 124 measured the strength of associations between multiple risk factors and the presence of parasite 125 larvae in cat's feces. 


\section{Results and discussion}

\subsection{Characteristics of the cat population}

130 A total of 950 cats were examined with ages ranging from 2 to 240 months (mean age 53.1

$131 \pm 37.7$ months). Approximately, 53.2\% (506) of the cats were females and $46.7 \%$ (444) were 132 males. Breed distribution included 910 (95.78\%) domestic short hair, 27 (2.84\%) domestic 133 longhair and $13(1.36 \%)$ belonged to British Semi longhair $(n=6)$, British longhair $(n=3)$, 134 Bengal $(n=1)$, Burmese $(n=1)$, Cornish Rex $(n=1)$, and Maine Coon $(n=1)$.

\subsection{Prevalence of and risk factors associated with infection}

In our study, $1.7 \%(17 / 950)$ of the fecal samples tested were positive. According to a recent epidemiological survey conducted across 12 European countries, feline lungworms were the second most frequent group of nematodes diagnosed in cats, and $A$ abstrusus was the most

140 frequently detected lungworm species across Europe, but none of the samples tested from cats

141 in Cambridge (UK) was positive for A. abstrusus (Giannelli et al., 2017). However, the 0\% prevalence reported previously from Cambridge may not be representative of the cat population in The UK due to the very small sample size examined. In fact, prevalence obtained in our study seems to fall within the prevalence range reported in Europe, which varied greatly from

$1450.38 \%$ in Croatia (Grabarević et al., 1999), 1\% in Germany (Mundhenke and Daugschies, 146 1999) 2.08\% in Ireland (Garcia-Campos et al., 2018), 2.3\% in Switzerland (Zottler et al., 2019),

$1472.6 \%$ in the Netherlands (Robben et al., 2004), 8.3\% in the Denmark (Hansen et al., 2017), $14826.5 \%$ in in Italy (Genchi et al., 2014), to 43.1\% in Albania (Knaus et al., 2011).

149 The prevalence rate of $A$. abstrusus can also vary significantly within the same country, 150 for example in Denmark local prevalence rates varied from 0\% [95\% CI: $0.0-8.8$ ] to 31.4\% 
151 [95\% CI: 16.9-49.3] (Hansen et al., 2017). Difference in prevalence rates was also detected 152 among three regions in Italy (Giannelli et al., 2017). A similar trend was detected in our study

153 where significant differences were observed in the geographic regions in regard to their 154 association with the increase in the odds of $A$. abstrusus infection, in comparison with East 155 Midlands region (Table 1). The disparity among the prevalences of feline lungworm $A$. 156 abstrusus across geographic regions may reflect the level of transmission or availability of 157 intermediate and paratenic reservoir hosts that are able to maintain $A$. abstrusus life cycle in 158 certain areas. The broad geographic distribution of $A$. abstrusus in our study indicates that $A$. 159 abstrusus is circulating in cat population and not restricted to a certain locality in England.

160 Further epidemiological studies are required to determine the factors that drive the transmission 161 of A. abstrusus in the areas where this parasite is highly prevalent. In this study, the larvae per gram of feces (LPG) were determined using Baermann's technique and ranged from 8 to $22(11.6 \pm 3.3)$. This was surprisingly low compared to a 164 previous study that detected a mean of 508.7 LPG (Giannelli et al., 2017). The larval survival 165 tend to decline, due to dehydration, depending on the cat litter type and the duration of time 166 faecal samples remain in the litter; a reduction in the viability of $80 \%$ of larvae occurred after $1673 \mathrm{~h}$ and reached almost 100\% after $24 \mathrm{~h}$ (Abbate et al., 2018). In our study, the length of time samples remained in the litter before collection ranged from 1 to $4 \mathrm{~h}$. Thus, we must be 169 cognizant of the potential influence of dehydration on the larval viability, given the low170 parasitic load and the time elapsed while the samples are present in the cat litter, which may 171 have underestimated the isolation rate of larvae in our study. It is also worth mentioning that 172 although Baermann's technique is specifically used for direct isolation of lungworm larvae 173 from feces, its diagnostic performance and sensitivity can be compromised by various factors.

174 These include the inability to isolate larvae in the pre-patent period, inconsistent shedding of 175 the larvae especially in cases with low parasite burdens, or cessation of shedding larvae by 
176 some cats, despite being infected, which in turn lead to false negative results (Hamilton, 1968;

177 Elsheikha et al., 2016). To increase the accuracy of detection of $A$. abstrusus in future surveys,

178 Baermann's technique should be performed on freshly voided fecal samples collected on three

179 consecutive days. Serological detection of antibodies has dramatically improved the sensitivity

180 of detection of lungworms (Zottler et al., 2017), adding more value for the diagnosis of feline

181 aelurostrongylosis. Thus, a greater emphasis on the use of a serological assay in conjunction

182 with fecal analysis may be warranted to achieve more accurate laboratory diagnosis.

183 We examined the association between age, breed, gender, lifestyle, deworming history,

184 and the risk of infection with $A$. abstrusus. Our risk assessment analysis did not detect any

185 effect of the age or breed on the frequency of infection. However, the risk of $A$. abstrusus

186 infection in Denmark was lower in kittens younger than 11 weeks compared to older cats

187 (Hansen et al., 2017). Another study reported significantly higher prevalence in cats younger

188 than 2 years and in cats co-infected with other gastrointestinal parasites (Giannelli et al., 2017).

189 In the present and previous studies, gender was not a risk factor for infection with $A$. abstrusus

190 (Traversa et al., 2008; Barutzki and Schaper, 2013; Olsen et al., 2015; Hansen et al., 2017).

191 Also, we did not detect any differences between neutered and intact cats

192 Out of the 17 infected cats, 13 had outdoor access, three were stray cats and one was an

193 indoor cat (Table 2). Keeping cats indoor was associated with significant protection against

194 infection (odds ratio $[\mathrm{OR}]=0.09 ; 95 \%$ confidence interval $[\mathrm{CI}]=0.01$ to $0.48 ; p=0.02$ ). In

195 contrast, no significant difference was detected between stray cats and cats with outdoor access

$196(\mathrm{OR}=1.01 ; 95 \% \mathrm{CI}=0.22$ to $3.49 ; p=0.99)$; probably due to the small number of cats in

197 these categories. These findings lend further support to previously reported findings where

198 rural origin, feral lifestyle and outdoor access have been shown to correlate with an increased

199 risk of infection (Traversa et al., 2009; Beugnet et al., 2014; Hansen et al., 2017). 
Out of the 17 infected cats, four cats were treated with various anthelmintics on the day of sample collection and 13 cats were non-treated. Our analysis has shown a lack of correlation between deworming history and risk of infection. Although deworming was not associated with a significant reduction in the infection risk, treatment is still a key factor that influences the

204 frequency of infection with lungworm in cats. This is because many cats with outdoor access 205 have more opportunity to acquire infection by preying on intermediate and transport hosts. 206 Also, the frequency and timing of deworming are likely to affect the likelihood of infection.

207 Additionally, these results should be interpreted with caution given the small number of 208 positive samples in the stratified categories, which may not have been sufficient to identify any 209 protective effect of deworming.

210 In conclusion, the present study addressed a significant aspect of the epidemiology of $A$.

211 abstrusus, a potential serious health problem in feline medicine. Our findings demonstrate that

212 A. abstrusus is present in $1.7 \%$ of cats in England, and infection frequency seems to vary 213 according to the geographic region and lifestyle. These findings suggest that $A$. abstrusus 214 should be considered a potential cause of respiratory tract disease in cats presenting with 215 pulmonary manifestations including cats with mild respiratory signs. However, it is possible 216 that cats can be infected and shed high number of larvae in feces without presenting clear 217 clinical signs. Therefore, integrated strategies for management of $A$. abstrusus as well as other 218 feline metastrongyloid lungworms (Troglostrongylus spp., Oslerus rostratus, Capillaria 219 aerophila), should be implemented and can be achieved through using preventative anthelmintics, enhanced diagnostics and increased awareness of feline lungworms. To this end, awareness and education campaigns, launched by pharma and professional organizations, such as European Scientific Counsel Companion Animal Parasites (ESCCAP) and Companion

223 Animal Parasite Council (CAPC), which promote adherence to lungworm prophylaxis should 224 be tailored to at-risk cat populations. 


\section{Acknowledgements}

The authors are grateful for the support of Cat protection, local catteries and private practices for providing the fecal samples. The study was kindly funded by Bayer Animal

Health. We are grateful for the excellent technical assistance of Paul Goodwin (University of

230 Nottingham).

\section{References}

Abbate, J.M., Arfuso, F., Gaglio, G., Napoli, E., Cavalera, M.A., Giannetto, S., Otranto, D., Brianti, E., 2018. Larval survival of Aelurostrongylus abstrusus lungworm in cat litters. J Feline Med Surg. Nov 12:1098612X18811168. doi: 10.1177/1098612X18811168. [Epub ahead of print]

Barutzki, D., Schaper, R., 2013. Occurrence and regional distribution of Aelurostrongylus abstrusus in cats in Germany. Parasitol. Res. 112, 855-861.

Beugnet, F., Chalvet-Monfray, K., Cozma, V., Farkas, R., Guillot, J., Halos, L., Joachim, A., Losson, B., Miro, G., Otranto, D., Renaud, M., Rinaldi, L., 2014. Parasites of domestic owned cats in Europe: co-infestations and risk factors. Parasit. Vectors 7,: 291.

Brianti, E., Giannetto, S., Dantas-Torres, F., Otranto, D., 2014. Lungworms of the genus Troglostrongylus (Strongylida: Crenosomatidae): neglected parasites for domestic cats. Vet. Parasitol. 202, 104-112.

Elsheikha, H.M., Schnyder, M., Traversa, D., Di Cesare, A., Wright, I., Lacher, D.W., 2016. Updates on feline aelurostrongylosis and research priorities for the next decade. Parasit. Vectors. 9(1), 389. 
249 Elsheikha, H.M., Schunack, B., Schaper, R., 2017. Prevalence of feline lungworm 250 Aelurostrongylus abstrusus in England. Abstract Book , 407, The 26th International 251 Conference of the World Association for the Advancement of Veterinary Parasitology, WAAVP, Kuala Lumpur, Malaysia.

Garcia-Campos, A., Power, C., O'Shaughnessy, J., Browne, C., Lawlor, A., McCarthy, G., O'Neill, E.J., de Waal, T., 2018. One-year parasitological screening of stray dogs and cats in County Dublin, Ireland. Parasitology. Dec 18:1-7. doi: 10.1017/S0031182018002020. [Epub ahead of print]

Genchi, M., Ferrari, N., Fonti, P., De Francesco, I., Piazza, C., Viglietti, A., 2014. Relation between Aelurostrongylus abstrusus larvae excretion, respiratory and radiographic signs in naturally infected cats. Vet. Parasitol. 206 (3/4), 182-187.

Gerichter, C.B., 1949. Studies on the nematodes parasitic in the lungs of Felidae in Palestine. Parasitology 39, 251-262.

Giannelli, A., Capelli, G., Joachim, A., Hinney, B., Losson, B., Kirkova, Z., René-Martellet, M., Papadopoulos, E., Farkas, R., Napoli, E., Brianti, E., Tamponi, C., Varcasia, A., Margarida, Alho A., Madeira de Carvalho, L., Cardoso, L., Maia, C., Mircean, V., Mihalca, A.D., Miró, G., Schnyder, M., Cantacessi, C., Colella, V., Cavalera, M.A., Latrofa, M.S., Annoscia, G., Knaus, M., Halos, L., Beugnet, F., Otranto, D., 2017. Lungworms and gastrointestinal parasites of domestic cats: a European perspective. Int. J. Parasitol. 47, 517-528.

Giannelli, A., Ramos, R.A., Annoscia, G., Di Cesare, A., Colella, V., Brianti, E., DantasTorres, F., Mutafchiev, Y., Otranto, D., 2014. Development of the feline lungworms Aelurostrongylus abstrusus and Troglostrongylus brevior in Helix aspersa snails. Parasitology 141, 563-569. 
Grabarević Ž., Ćurić S., Tustonja A., Artuković B., Šimec Z., Ramadan K., Živičnjak T. 1999. Incidence and regional distribution of the lungworm Aelurostrongylus abstrusus in cats in Croatia. Veterinarski Arhiv 69, 279-287.

Gunn-Moore, D., Elsheikha, H.M., 2018. Current status of feline lungworm in the UK. Vet. Rec. 182(4), 113-114.

Hamilton, J.M., 1968. Studies on re-infestation of the cat with Aelurostrongylus abstrusus. J. Comp. Pathol. 78(1), 69-72.

Hansen, A.P., Skarbye, L.K., Vinther, L.M., Willesen, J.L., Pipper, C.B., Olsen, C.S., Mejer, H., 2017. Occurrence and clinical significance of Aelurostrongylus abstrusus and other endoparasites in Danish cats. Vet. Parasitol. 234, 31-39.

Knaus, M., Kusi, I. Rapti, D., Xhaxhiu, D.,Winter, R., Visser, M., Rehbein, S., 2011. Endoparasites of cats from the Tirana area and the first report on Aelurostrongylus abstrusus (Railliet, 1898) in Albania. Wien. Klin. Wochenschr. 123, 31-35.

Mundhenke, H., Daugschies, A., 1999. Studies on the prevalence of endoparasites in cats in Hannover and surroundings. Wien. Tierarztl. Monatsschr. 86 (2), 43-48.

Olsen, C.S., Willesen, J.L., Pipper, C.B., Mejer, H.., 2015. Occurrence of Aelurostrongylus abstrusus (Railliet, 1898) in Danish cats: A modified lung digestion method for isolating adult worms. Vet. Parasitol. 210, 32-39.

Penagos-Tabares, F., Lange, M.K., Chaparro-Gutiérrez, J.J., Taubert, A., Hermosilla, C., 2018. Angiostrongylus vasorum and Aelurostrongylus abstrusus: Neglected and underestimated parasites in South America. Parasit. Vectors 11, 208.

Pennisi, M.G., Hartmann, K., Addie, D.D., Boucraut-Baralon, C., Egberink, H., Frymus, T., Gruffydd-Jones, T., Horzinek, M.C., Hosie, M.J., Lloret, A., Lutz, H., Marsilio, F., Radford, A.D., Thiry, E., Truyen, U., Möstl, K.; European Advisory Board on Cat 
Diseases., 2015. Lungworm disease in cats: ABCD guidelines on prevention and management. J. Feline Med. Surg. 17, 626-636.

Robben, S.R., le Nobel, W.E., Döpfer, D., Hendrikx, W.M., Boersema, J.H., Fransen, F., Eysker, M.E., Infections with helminths and/or protozoa in cats in animal shelters in the Netherlands.2004. Tijdschr Diergeneeskd. 129(1), 2-6.

Scott, D.W., 1973. Current knowledge of aelurostrongylosis in the cat. Literature review and case reports. Cornell Vet. 63, 483-500.

Traversa, D., Lia, R.P., Boari, A., Di Cesare, A., Capelli, G., Milillo, P., Otranto, D., 2009. Feline aelurostrongylosis: epidemiological survey in central and southern Italy. Veterinaria 23, 41-45.

Traversa, D., Lia, R.P., Iorio, R., Boari, A., Paradies, P., Capelli, G., Avolio, S., Otranto, D., 2008. Diagnosis and risk factors of Aelurostrongylus abstrusus (Nematoda, Strongylida) infection in cats from Italy. Vet. Parasitol. 153, 182-186.

Zottler, E.M., Strube, C., Schnyder, M., 2017. Detection of specific antibodies in cats infected with the lung nematode Aelurostrongylus abstrusus. Vet Parasitol. 235, 75-82. in stray, shelter and privately owned cats of Switzerland. Parasitol. Int. 69, 75-81. 
Table 1.

323 The prevalence of $A$. abstrusus across seven administrative regions in England.

\begin{tabular}{|c|c|c|c|c|}
\hline Geographic region & Prevalence* & Odd ratios & $95 \% \mathrm{CI}$ & $p$-value \\
\hline Greater London & $1 / 17(5.8)$ & 9.632 & 0.43 to 84.05 & 0.07 \\
\hline South East & $4 / 72(5.5)$ & 7.68 & 1.70 to 32.76 & 0.01 \\
\hline West Midlands & $3 / 69(4.3)$ & 6.2 & 1.21 to 26.84 & 0.02 \\
\hline North West & $2 / 57(3.5)$ & 4.25 & 0.59 to 20.89 & 0.09 \\
\hline South West & $1 / 46(2.1)$ & 2.48 & 0.12 to 17.64 & 0.43 \\
\hline North East & $1 / 49(2.0)$ & 1.88 & 0.10 to 12.24 & 0.57 \\
\hline
\end{tabular}

327

328

329

330

331

332

333

334

335

336

337

338

339 
340 Table 2.

341 Lifestyle distribution and positivity rates of $A$. abstrusus in cats examined in this study.

342 Correlation was established only between cats living indoor and A. abstrusus infection.

\begin{tabular}{llll}
\hline Lifestyle category & No. of & No. of & Larvae per \\
& uninfected cats & infected cats & gram of feces \\
\hline Indoor & 375 & 1 & 12.0 \\
Outdoor Access & 455 & 13 & 11.5 \\
Stray & 81 & 3 & 12.0 \\
Feral & 22 & 0 & 0.0 \\
Total $(n=950)$ & 933 & 17 & 11.6
\end{tabular}

343

344 